\title{
Case Report \\ Solitary Fibrous Tumor of the Kidney Developing Local Recurrence
}

\author{
Wataru Usuba, ${ }^{1}$ Hideo Sasaki, ${ }^{1}$ Hidekazu Yoshie, ${ }^{1}$ Kazuki Kitajima, ${ }^{1}$ Hiroya Kudo, ${ }^{1}$ \\ Ryuto Nakazawa, ${ }^{1}$ Yuichi Sato, ${ }^{1}$ Masayuki Takagi, ${ }^{2}$ and Tatsuya Chikaraishi ${ }^{1}$ \\ ${ }^{1}$ Department of Urology, St. Marianna University, 2-16-1 Sugao, Miyamae, Kawasaki 216-8511, Japan \\ ${ }^{2}$ Department of Pathology, St. Marianna University, 2-16-1 Sugao, Miyamae, Kawasaki 216-8511, Japan \\ Correspondence should be addressed to Wataru Usuba; wataruusuba0319@gmail.com
}

Received 21 January 2016; Revised 30 March 2016; Accepted 7 April 2016

Academic Editor: Phillip M. Pierorazio

Copyright (C) 2016 Wataru Usuba et al. This is an open access article distributed under the Creative Commons Attribution License, which permits unrestricted use, distribution, and reproduction in any medium, provided the original work is properly cited.

Solitary fibrous tumor (SFT) of the kidney is a rare entity and usually displays a favorable prognosis. We herein report a second case of renal SFT developing local recurrence. A 50-year-old man was referred to our hospital because of a left renal mass. An abdominal CT detected a large renal tumor and radical nephrectomy was performed with a possible diagnosis of renal cell carcinoma. The resected tumor size was measured at $17 \times 11 \times 8 \mathrm{~cm}$. Grossly, necrosis was observed in central lesion of the tumor but hemorrhage was not observed. Microscopically, the tumor consisted of spindle-shaped cells with scant cytoplasm accompanied by hyalinized collagenous tissue, which displayed hemangiopericytomatous patterns. The cellularity was normal and nuclear pleomorphism was not observed. Ki-67 labeling index was less than 3\%. The pathological diagnosis of SFT was made without obvious malignant findings. Three years after the surgery, a follow-up CT scan detected a mass lesion in the tumor bed. Surgical resection was performed and the resected tumor was compatible with local recurrence of the SFT without obvious malignant findings. Renal SFT should be carefully monitored even in the absence of obvious malignant findings.

\section{Introduction}

Solitary fibrous tumor (SFT) is a clinical entity that was first reported as a tumor of the pleura in 1931 and usually arises in the pleura [1]. SFT is a rare spindle cell neoplasm and it is postulated that the tumor originated from mesenchymal tissue [2]. Histologically SFT shows hemangiopericytomalike growth pattern and immunohistochemical staining for CD-34 and Bcl-2 is helpful for diagnosing the SFT. SFT typically is strong and diffusely positive for CD-34 and 70\% of the SFT is positive for Bcl-2 [3]. The disease commonly arises from the thoracic cavity, yet it may arise from other sites including the kidney [2]. SFT of the kidney is an extremely rare and generally indolent tumor, unlikely to recur locally or distantly. Up to the present, only 81 cases of occurring renal SFT have been reported. SFT of the kidney usually displays a favorable prognosis and only two cases were reported to develop a distant metastasis. Furthermore local recurrence of SFT of the kidney had been reported in only one case [4].
Herein, we describe the second case of local recurrence of renal SFT after radical.

\section{Case Presentation}

A 50-year-old male was referred to our hospital because of a left renal mass, which had been incidentally detected by ultrasonography performed in a routine health checkup. A physical examination and blood chemical analysis were normal. Subsequent computed tomography (CT) scan detected a well-enhanced large left renal tumor (Figure 1(a)). He was diagnosed with left renal cell carcinoma preoperatively, and radical nephrectomy was performed. Grossly, the tumor was measured at $17 \times 11 \times 8 \mathrm{~cm}$, was wellcircumscribed, and displayed necrosis with a gray-white cut surface. Hemorrhage was not observed. Microscopically, the tumor was composed of spindle-shaped cells, which displayed hemangiopericytomatous patterns (Figure 2(a)). The tumor displayed normal cellularity without nuclear 


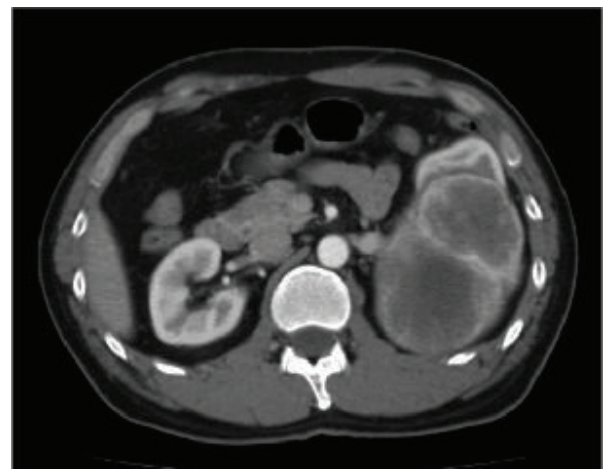

(a)

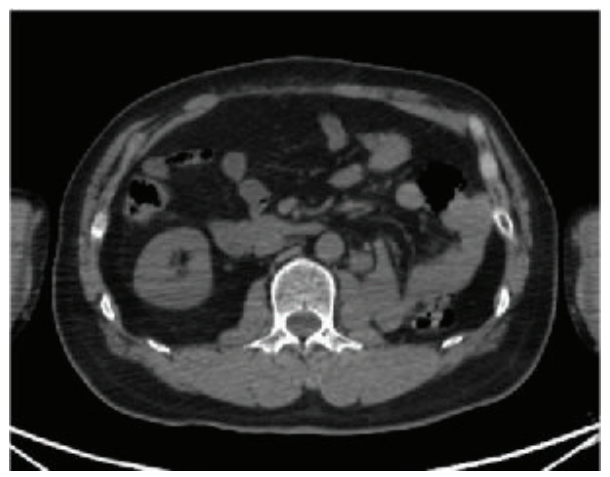

(c)



(b)

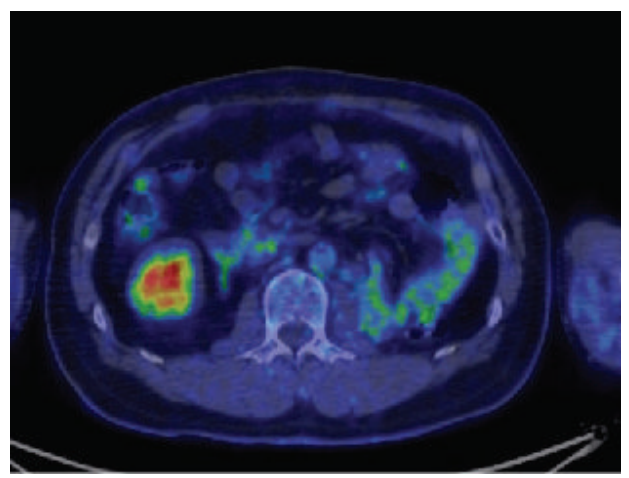

(d)

FIGURE 1: Radiological findings of the renal SFT. Enhanced abdominal CT revealed $17 \times 11 \times 8 \mathrm{~cm}$ tumor located in the left kidney (a). Followup plain CT revealed suspicions of recurrent tumor $(1 \times 0.7 \mathrm{~cm})$ in the tumor bed at 3 years after the nephrectomy (b). Three months after the CT, which detected suspicions of recurrent tumor, follow-up CT scan and PET-CT were performed. (c, d) The mass lesion was increased in size $(1.7 \times 1.1 \mathrm{~cm})$.

pleomorphism. Mitotic count was less than 1 per 10 high power fields. Immunohistochemical staining was positive for CD-34 (Figure 2(b)), Bcl-2 (Figure 2(c)), CD-99, and STAT-6, all of them representing conventional immunohistochemical markers for SFT. Meanwhile, SMA stain was negative and Ki67 labeling index was less than 3\% (Figure 2(d)). Thus, he was histologically diagnosed with SFT of the kidney without obvious malignant findings. Postoperatively, follow-up CT examination was performed regularly every 3-4 months. Three years after the operation, a mass lesion was detected in the tumor bed (Figure 1(b)). The mass lesion was increased in size after 3 months (Figure 1(c)). Fluorodeoxyglucose (FDG) positron emission tomography (PET) was ordered but the tumor did not accumulate FDG (Figure 1(d)). Nonetheless, as a local recurrence or lymph node metastasis could not be denied, we planned a surgical removal of the tumor. Although the recurrent tumor displayed spindleshaped cells with hemangiopericytomatous patterns as in the original tumor, the cellularity was increased and cytological atypia was observed (Figure 2(e)). These results suggested an increased malignant potential of the tumor, but mitotic count was less than 4 mitoses per 10 high power fields. Immunohistochemical staining for CD-34 (Figure 2(f)), Bcl2 (Figure 2(g)), and CD-99 all remained positive. Ki-67 labeling index was less than 15\% (Figure 2(h)) and SMA stain was positive in the resected tissue from the tumor bed. Although an increased malignant potential was suggested, pathological findings did not meet the diagnostic criteria of malignant SFT [5]. The recurrent tumor was developed from an extra nodal connective tissue not from the lymph node (Figure 3). Therefore, we diagnosed local recurrence of renal SFT without evidence of obvious malignant findings. Twelve months after the second operation, the patient is followed up on the outpatient basis with no evidence of local recurrence or distant metastasis.

\section{Discussion}

In 1931, SFT was firstly reported as a tumor of the pleura [1]. It is a rare tumor comprising spindle-shaped cells, which might originate from mesenchymal tissue [2]. Although SFT is commonly thought of as an intrathoracic tumor, it could arise from extrathoracic organs, including the kidney [2]. Surgical resection is a standard treatment and complete resection can be associated with a favorable prognosis, even if the SFT is histologically diagnosed as malignant $[4,6]$.

SFT of the kidneys is a rare neoplasm, and Sasaki et al. reviewed the 68 cases of SFT in 2013 [7], and additional 13 cases were reported up to now. All reported cases, including our case, are summarized in Table 1. Most of the tumors 


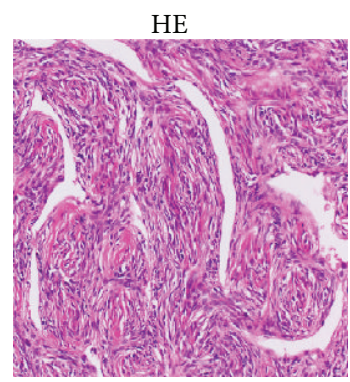

(a)

HE

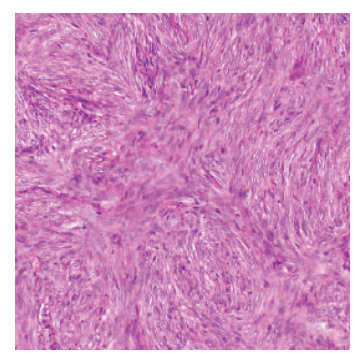

(e)

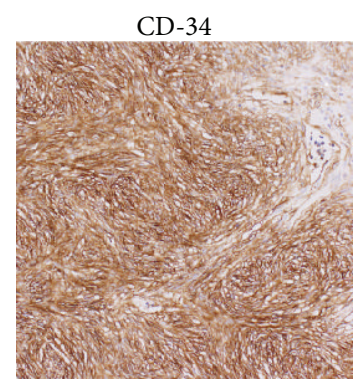

(b)

CD-34

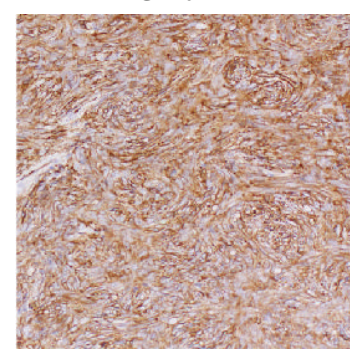

(f)

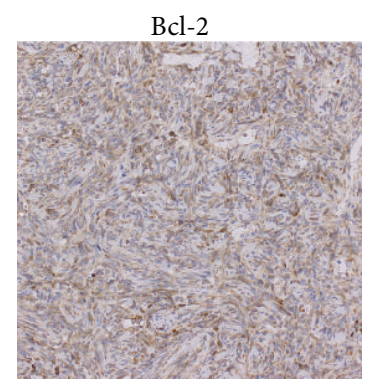

(c)

Bcl-2



(g)

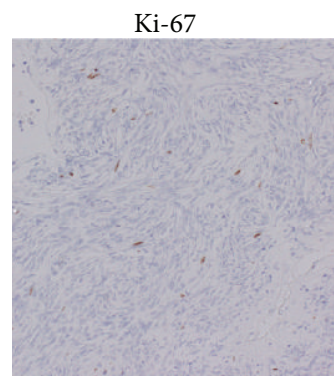

(d)

Ki-67

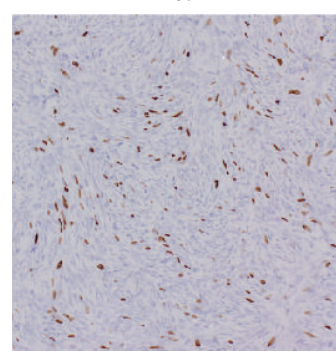

(h)

FIGURE 2: Histological findings of the solitary fibrous tumor. The primary tumor displayed hemangiopericytomatous patterns $((\mathrm{a}), \mathrm{HE} \times 40)$. Immunohistochemical staining of the primary tumor was positive for CD-34 and Bcl-2 ((b) and (c), $\times 40)$ and Ki-67 labeling index was less than $3 \%((\mathrm{~d}), \times 40)$. Cellularity was increased in the tumor that recurred at the hilar portion of the kidney (e). Immunohistochemical staining for CD-34 and Bcl-2 was positive ((f) and $(\mathrm{g}), \times 40)$. Ki-67 labeling index was less than $15 \%((\mathrm{~h}), \times 40)$.

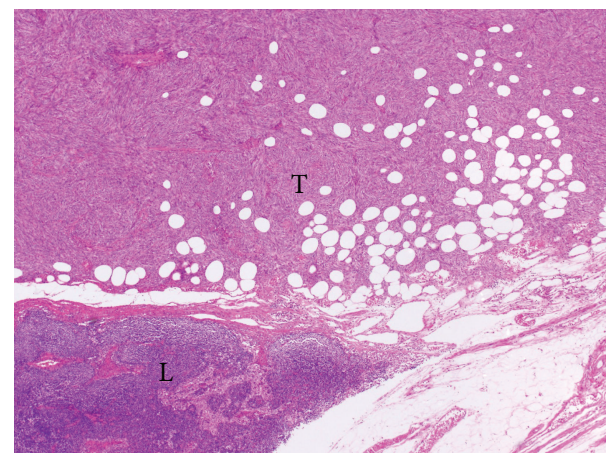

FIGURE 3: Histological finding of local recurrence of the solitary fibrous tumor. The recurrent tumor was developed from an extra nodal connective tissue $(\times 10)$. L: lymph node; T: tumor.

were incidentally found with no apparent clinical symptoms. Preoperatively, most of them were diagnosed as renal cell carcinoma, and 72 out of 82 cases underwent radical nephrectomy. Mean age at diagnosis was $52.8 \pm 17.7$ (3-85) years and mean tumor size was $9.5 \pm 6.2(2-29) \mathrm{cm}$. Histologically, 68 tumors showed a benign appearance, whereas 11 cases exhibited a malignant one. Most patients displayed a favorable prognosis with no evidence of recurrence during the follow-up period, ranging from 0.1 to 96 months. Only 4 patients experienced recurrence; 2 patients developed distant metastasis; and 2 patients, including the present case, developed local recurrence.
As SFT commonly expresses CD-34, Bcl-2, and CD-99 [8], these surface antigens can serve as useful diagnostic markers [8]. And negativity in CD-34 and Bcl-2 reportedly represents increased malignant potential $[8,9]$. Fine et al. documented a case of malignant renal SFT without expressing CD-34, which developed distant metastasis four months after surgery [10]. We also reported a similar case previously, which did not express CD-34 and went on to metastasize to the lung and liver [7]. In that case, half of the cross section area of the primary tumor was positive for CD-34, while the remaining area was negative for it. The patient developed distant metastases 8 years after nephrectomy. Resection of the metastatic tumors had revealed that CD-34 was totally absent in the tumors. Thus, the loss of CD-34 staining in SFT of the kidney may promote tumor metastasis to other organs [7]. Similarly to CD-34 staining, Bcl-2 staining was commonly observed in SFT and the loss of Bcl-2 staining was reported to be associated with malignant potential in retroperitoneal SFTs [9].

On the contrary, malignant potential is rather low in the present case, which developed local recurrence 3 years after nephrectomy. In this case, no obvious malignant findings were observed in either primary or recurrent tissue from the tumor bed. Furthermore, CD-34 and Bcl-2 were positive in the primary tumors and remained positive in the recurrent tissue. It seems that the local recurrence does not necessarily accompany the loss of expression of CD-34 and Bcl-2, and another explanation for unpredicted local recurrence would be incomplete resection at surgery [5]. However, from a different standpoint, the tumor in the present case may 


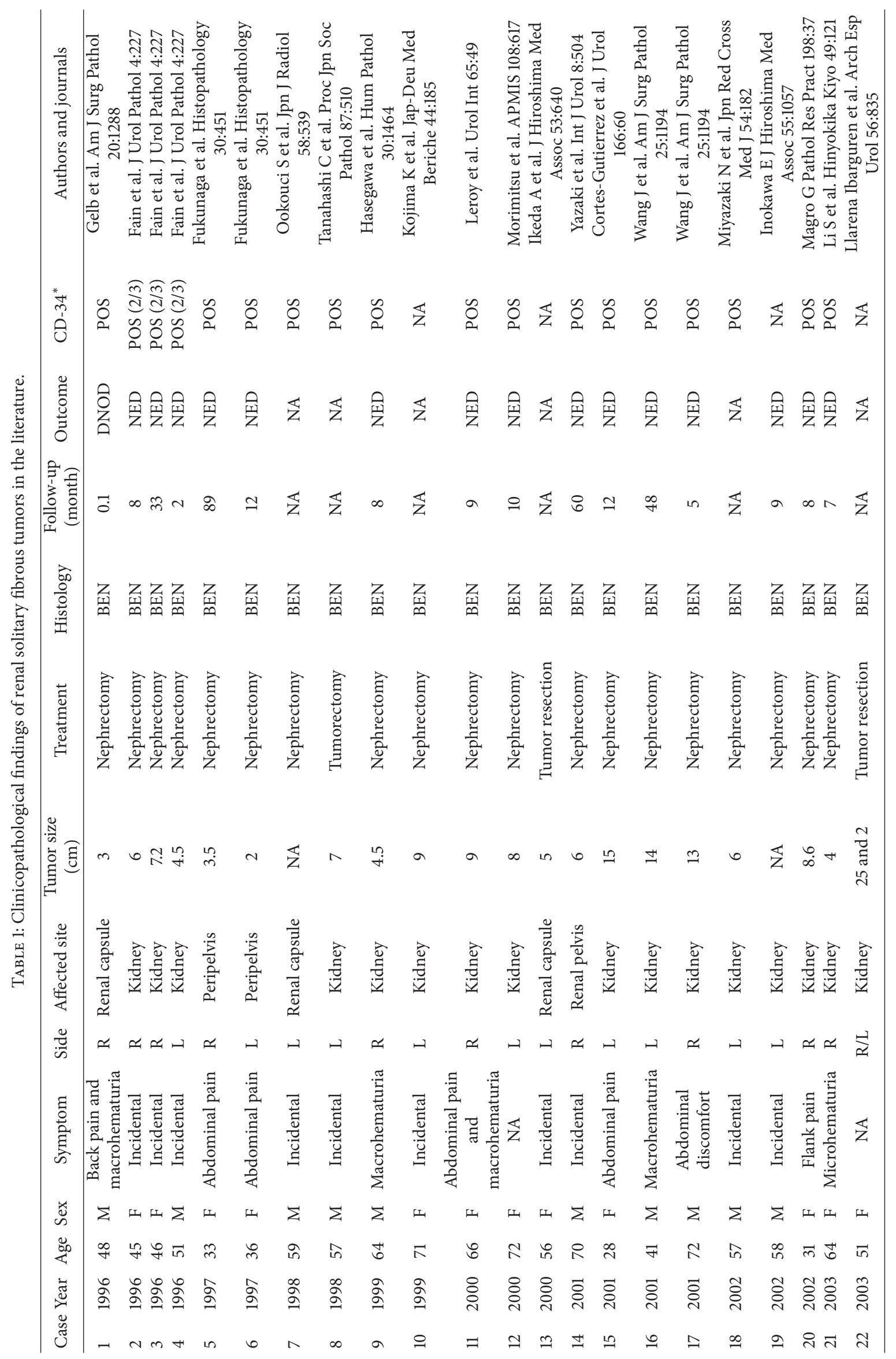




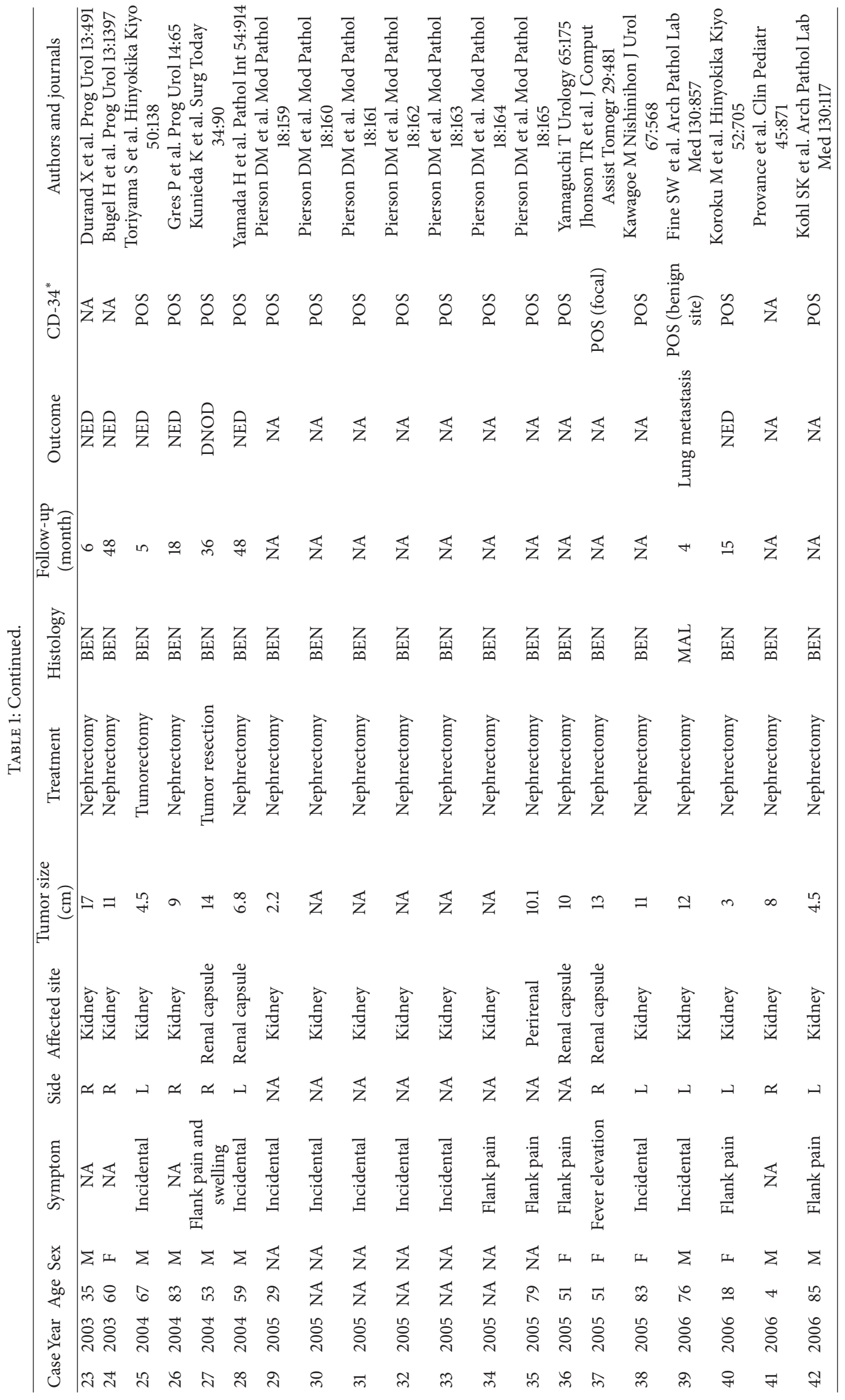




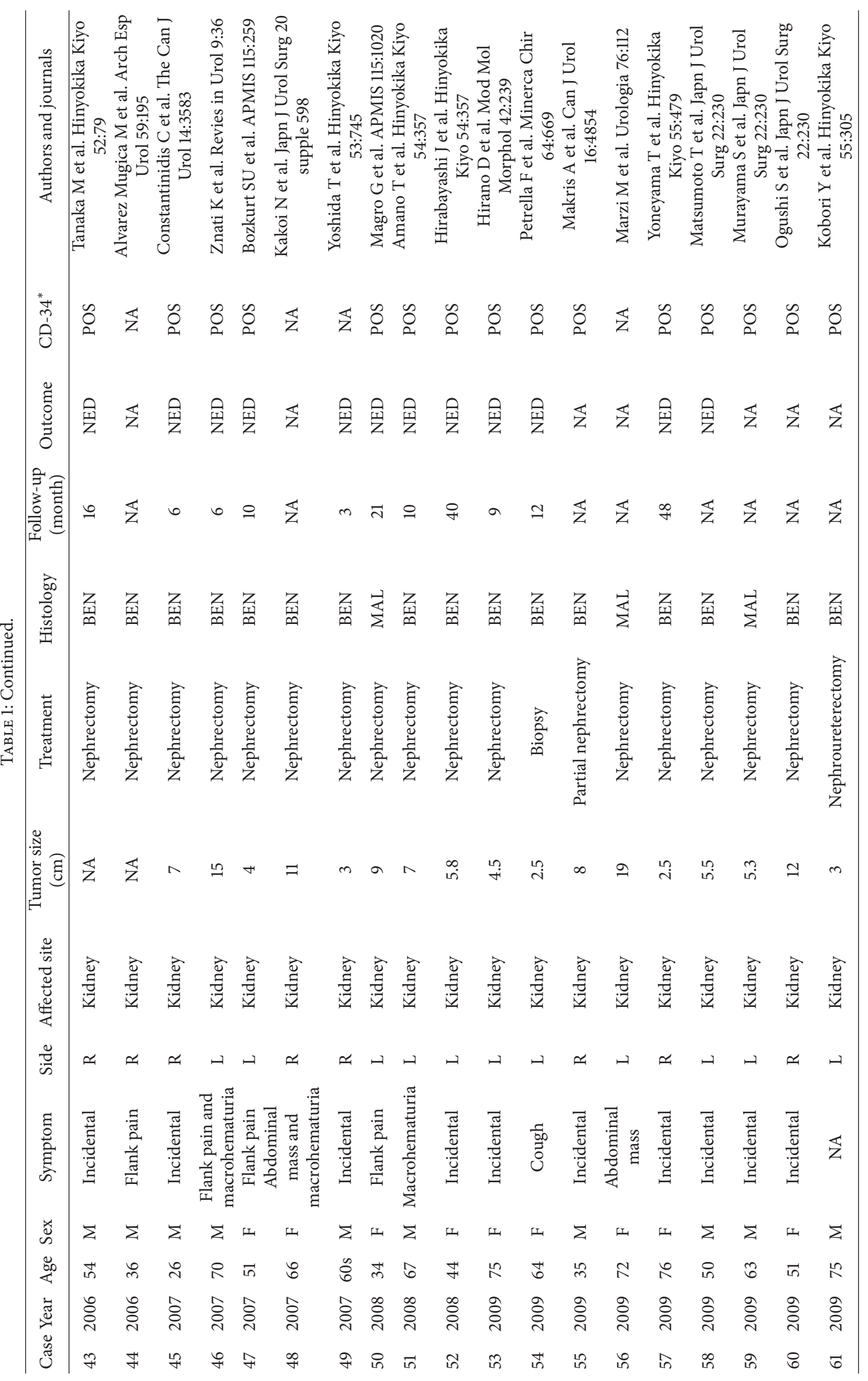




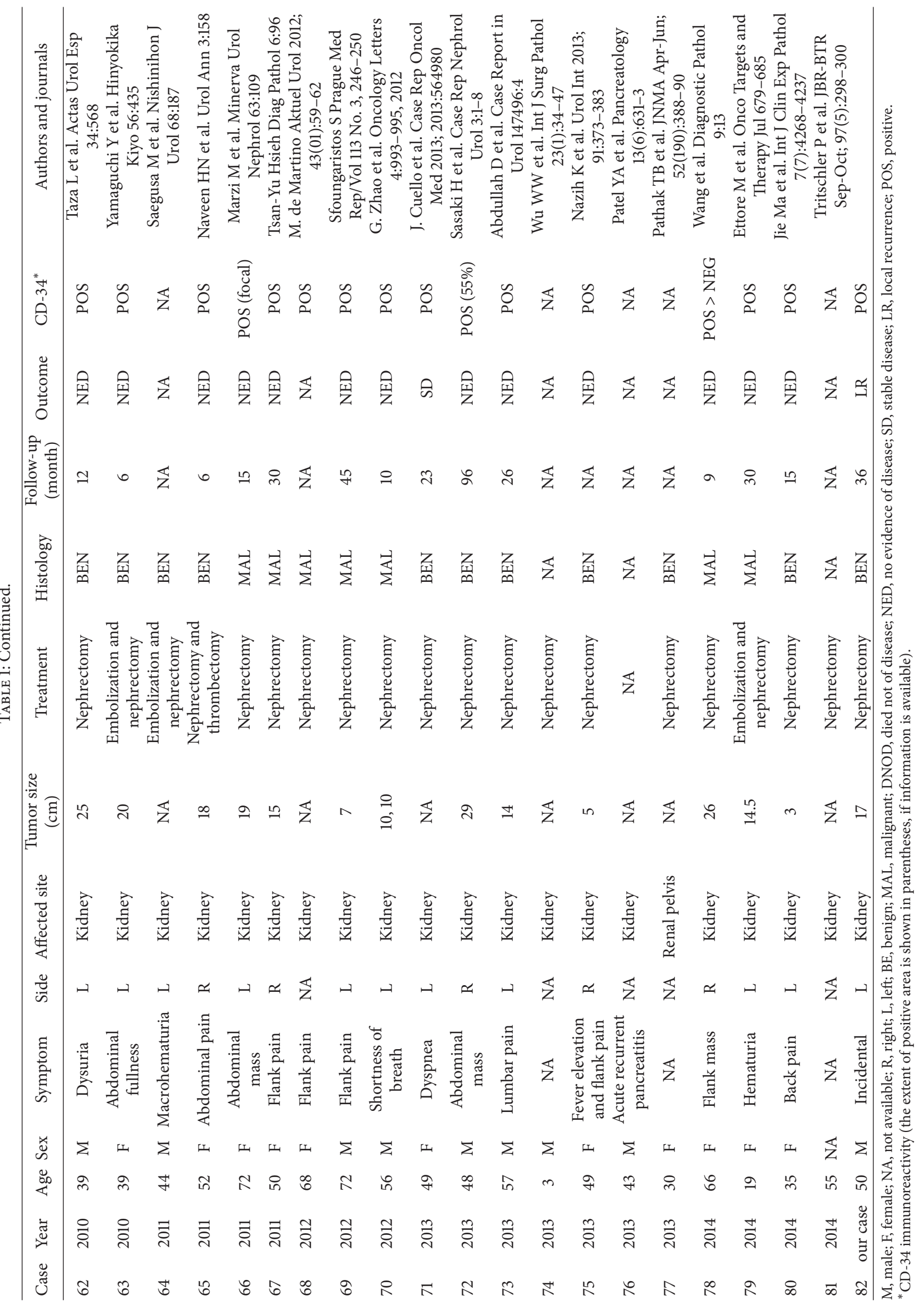


have had a great tendency to local recurrence, as the tumor accompanies multiple clinical features such as extrathoracic location, large tumor size, increased cellularity, and presence of necrosis among the risk factors for local recurrence described by Jason et al. [11].

Overall, we believe that there is no strict dichotomy between benign and malignant SFTs and that all tumors likely have some degree of metastatic potential, albeit quite low. Therefore, although renal SFT is thought to be a benign tumor, an adequate follow-up period is required to evaluate the precise clinical outcome of renal SFT, and the followup period in this report of 82 patients may not be sufficient (Table 1). Furthermore, most reported renal SFTs were large in size at the diagnosis and it might be leading cause of missing the malignant features in whole tumor tissue. We should also concern this issue for evaluating the real feature of renal SFTs in future.

FDG accumulation was not observed within the tumor on FDG-PET. To date, there is no reported association between SFTs and FDG accumulation, and our result suggests that PET-CT may be invalid. Further detailed examination is also required to clarify this point.

In conclusion, a case of SFT of the kidney exhibiting local recurrence was reported. In our case, no obvious malignant findings were observed in either the primary tumor or the recurrent tumor. Loss of expression in CD-34 and Bcl-2, which is closely associated with malignant potential, was not observed. Although SFT of the kidney usually displays a favorable clinical course, careful and sufficient follow-up may be required even in the absence of malignant findings.

\section{Consent}

The patient described in the case report has given his informed consent for the case report to be published.

\section{Competing Interests}

The authors declare that there is no conflict of interests regarding the publication of this paper.

\section{References}

[1] P. Klemperer and C. B. Rabin, "Primary neoplasm of the pleura: a report of five cases," Archives of Pathology \& Laboratory Medicine, vol. 11, pp. 385-412, 1931.

[2] J. R. Goodlad and C. D. M. Fletcher, "Solitary fibrous tumour arising at unusual sites: analysis of a series," Histopathology, vol. 19, no. 6, pp. 515-522, 1991.

[3] G. T. MacLennan and L. Cheng, "Solitary fibrous tumor of the kidney," Journal of Urology, vol. 181, no. 6, pp. 2731-2732, 2009.

[4] S. Sfoungaristos, M. Papatheodorou, A. Kavouras, and P. Perimenis, "Solitary fibrous tumor of the kidney with massive retroperitoneal recurrence. A case presentation ," Prague Medical Report, vol. 113, no. 3, pp. 246-250, 2012.

[5] D. M. England, L. Hochholzer, and M. J. McCarth, "Localized benign and malignant fibrous tumors of the pleura. A clinicopathologic review of 223 cases," The American Journal of Surgical Pathology, vol. 13, no. 8, pp. 640-658, 1989.
[6] Y. Morimitsu, M. Nakajima, M. Hisaoka, and H. Hashimoto, "Extrapleural solitary fibrous tumor: clinicopathologic study of 17 cases and molecular analysis of the p53 pathway," APMIS, vol. 108, no. 9, pp. 617-625, 2000.

[7] H. Sasaki, T. Kurihara, Y. Katsuoka et al., "Distant metastasis from benign solitary fibrous tumor of the kidney," Case Reports in Nephrology and Urology, vol. 3, no. 1, pp. 1-8, 2013.

[8] T. Yokoi, T. Tsuzuki, Y. Yatabe et al., "Solitary fibrous tumour: significance of p53 and CD34 immunoreactivity in its malignant transformation," Histopathology, vol. 32, no. 5, pp. 423-432, 1998.

[9] I. Takizawa, T. Saito, Y. Kitamura et al., "Primary solitary fibrous tumor (SFT) in the retroperitoneum," Urologic Oncology: Seminars and Original Investigations, vol. 26, no. 3, pp. 254-259, 2008.

[10] S. W. Fine, D. M. McCarthy, T. Y. Chan, J. I. Epstein, and P. Argani, "Malignant solitary fibrous tumor of the kidney: report of a case and comprehensive review of the literature," Archives of Pathology and Laboratory Medicine, vol. 130, no. 6, pp. 857-861, 2006.

[11] J. S. Gold, C. R. Antonescu, C. Hajdu et al., "Clinicopathologic correlates of solitary fibrous tumors," Cancer, vol. 94, no. 4, pp. 1057-1068, 2002. 


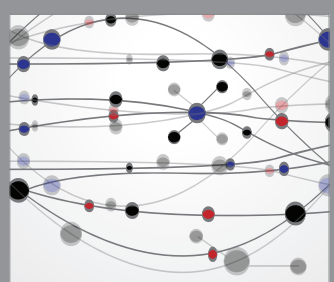

The Scientific World Journal
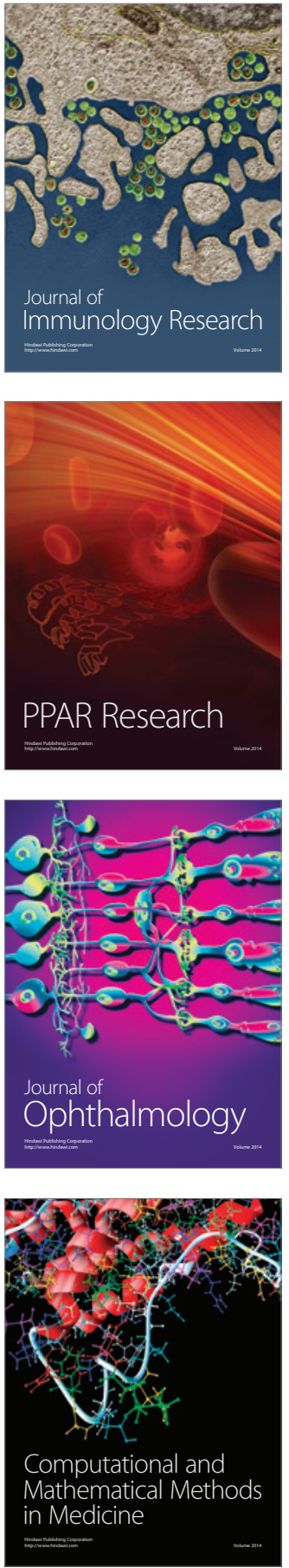

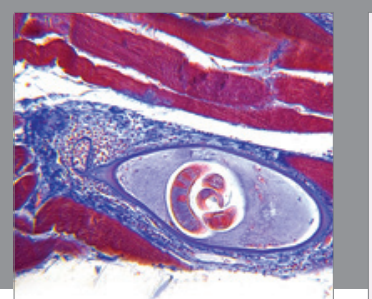

Gastroenterology Research and Practice

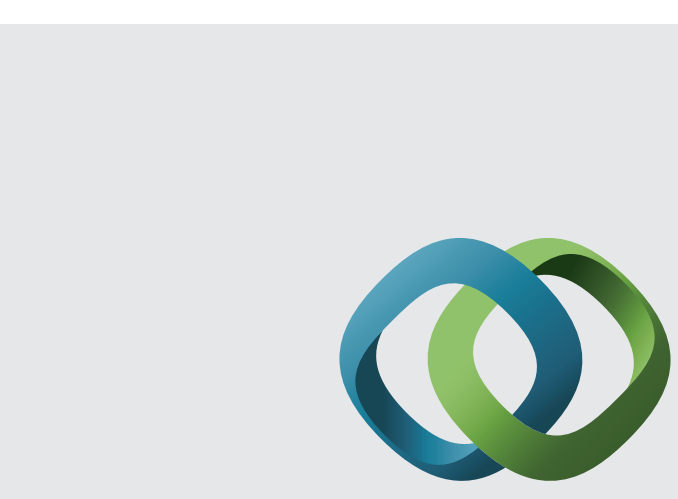

\section{Hindawi}

Submit your manuscripts at

http://www.hindawi.com
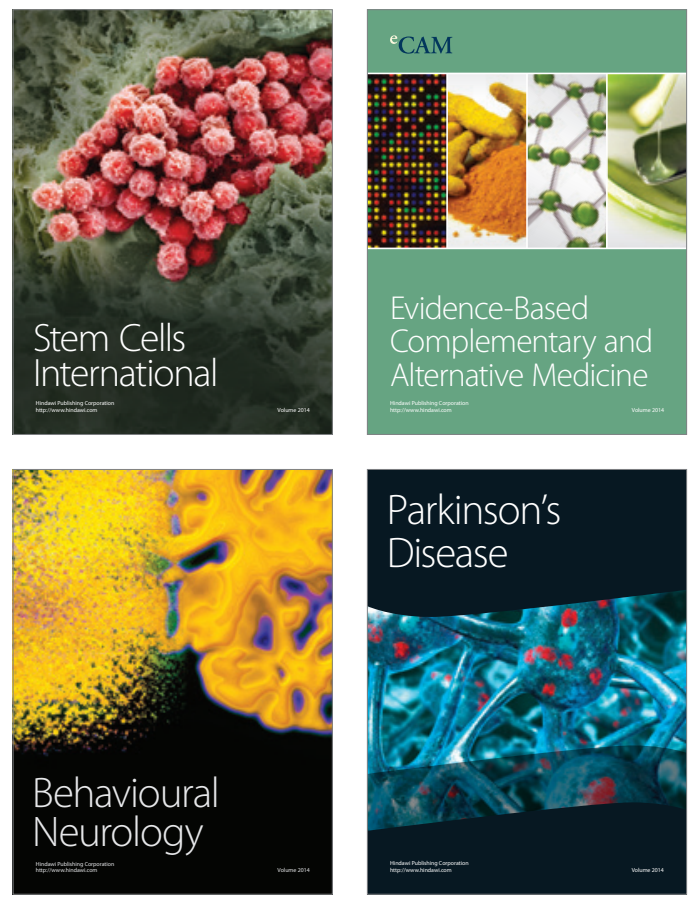
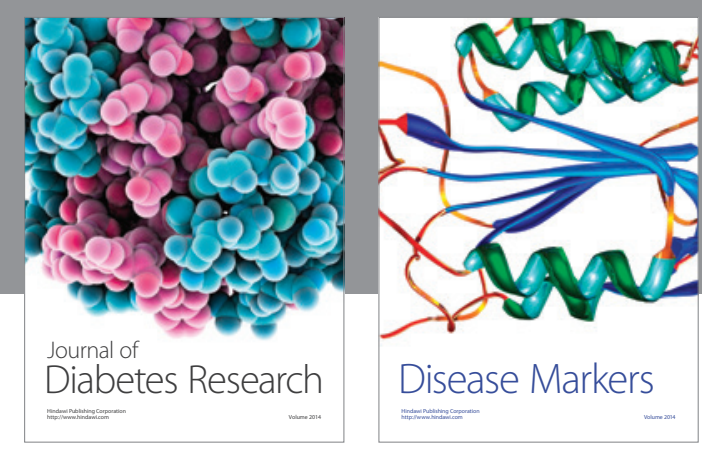

Disease Markers
